\title{
Interacting Cubane Assisted Bi-Cytidine with COVID-19 Main Protease: In Silico Study
}

\author{
Adil Muala Dhumad ${ }^{1(\mathbb{D})}$, Kun Harismah ${ }^{2, *}$ (I) , Hasan Zandi ${ }^{3(\mathbb{D})}$ \\ 1 Department of Chemistry, College of Education for Pure Sciences, University of Basrah, Basrah, Iraq; \\ adil.dhumad@uobasrah.edu.iq (A.M.D.); \\ 2 Department of Chemical Engineering, Faculty of Engineering, Universitas Muhammadiyah Surakarta, Surakarta, \\ Indonesia; kun.harismah@ums.ac.id (K.H.); \\ 3 Department of Chemistry, Faculty of Science, University of Qom, Qom, Iran; hasan_zandi@yahoo.com (H.Z.); \\ * Correspondence: kun.harismah@ums.ac.id;
}

Scopus Author ID 56982926300

Received: 22.01.2021; Revised: 19.02.2021; Accepted: 23.02.2021; Published: 2.03.2021

\begin{abstract}
In silico approach, the quantum chemical computations and molecular docking simulations have been used to investigate the formation of cubane assisted cytidine (B-Cyt) derivative for examining its interactions with the COVID-19 main protease. The obtained results indicated that the new B-Cyt derivative could be stabilized without any imaginary frequency. Its orbital orbital-based electronic properties indicated that the structure could have a better interaction with the target than the singular Cyt ligand. The docking process results approved the trend, in which the value of binding energy was very much favorable regarding the singular models, and the number of interaction amino acids was increased. The idea of forming a Cyt derivative with efficient activity against COVID-19 main protease was approved here, which is very much important for protecting the patients with cancer or HIV against the COVID-19 pandemic.
\end{abstract}

Keywords: cytidine; cubane; COVID-19; quantum computing; molecular docking.

(C) 2021 by the authors. This article is an open-access article distributed under the terms and conditions of the Creative Commons Attribution (CC BY) license (https://creativecommons.org/licenses/by/4.0/).

\section{Introduction}

Nucleobase derivatives have been seen as pharmaceutical compounds for several years with good potency and efficacy against different diseases but still could be improved [1-3]. Cytidine (Cyt) is among such derivatives with initial activity against cancer by inhibiting overexpressed enzymes [4]. In addition to the original Cyt, several other functionalized models have been developed to explore further features of Cyt for other types of pharmacotherapy [5-8]. Cyt itself is a pharmaceutical compound for cancer treatments, and earlier works have indicated that Cyt-related compounds could also work against HIV [9-12]. Since the Cyt compounds have been introduced as enzyme inhibitors, it is an important task to examine their activity against newly arisen ones such as the main protease of corona virus disease 19 (COVID-19) [13-15]. By the end of the year 2019, the COVID-19 pandemic has widely grown worldwide without and major therapeutic protocol yet, unfortunately. Therefore, it is an emergency case to innovate an efficient therapeutic protocol by drug design methodologies [16-18]. Several works have been dedicated to the topic in the last eight months, but further works are still required for the purpose [19-22]. The formation of a bi-cytidine (B-Cyt) compound with possible effects on the COVID-19 main protease was examined within this work. Cubane ( $\mathrm{Cub})$ trimer has been used as a molecular linker to make the possible formation 
of B-Cyt compound. Cub is a cubic structure of eight carbon atoms with its characteristic properties as a small stable hydrocarbon structure [23]. Moreover, several other functionalized models of Cub have been developed [24]. Chains of Cub have been seen as stable structures, in which Cub trimers have been seen as good connecting linkers for electronic devices [25]. Therefore, the idea of B-Cyt formation has been developed using the Cub trimer liker to combine two Cyt molecules. The stabilities of molecular structures have been examined using the quantum chemical computations (Table 1 and Figure 1), and their corresponding effects on COVID-19 have been examined by molecular docking simulations (Table 2 and Figure 2) as advantages of working in silico [26-32].

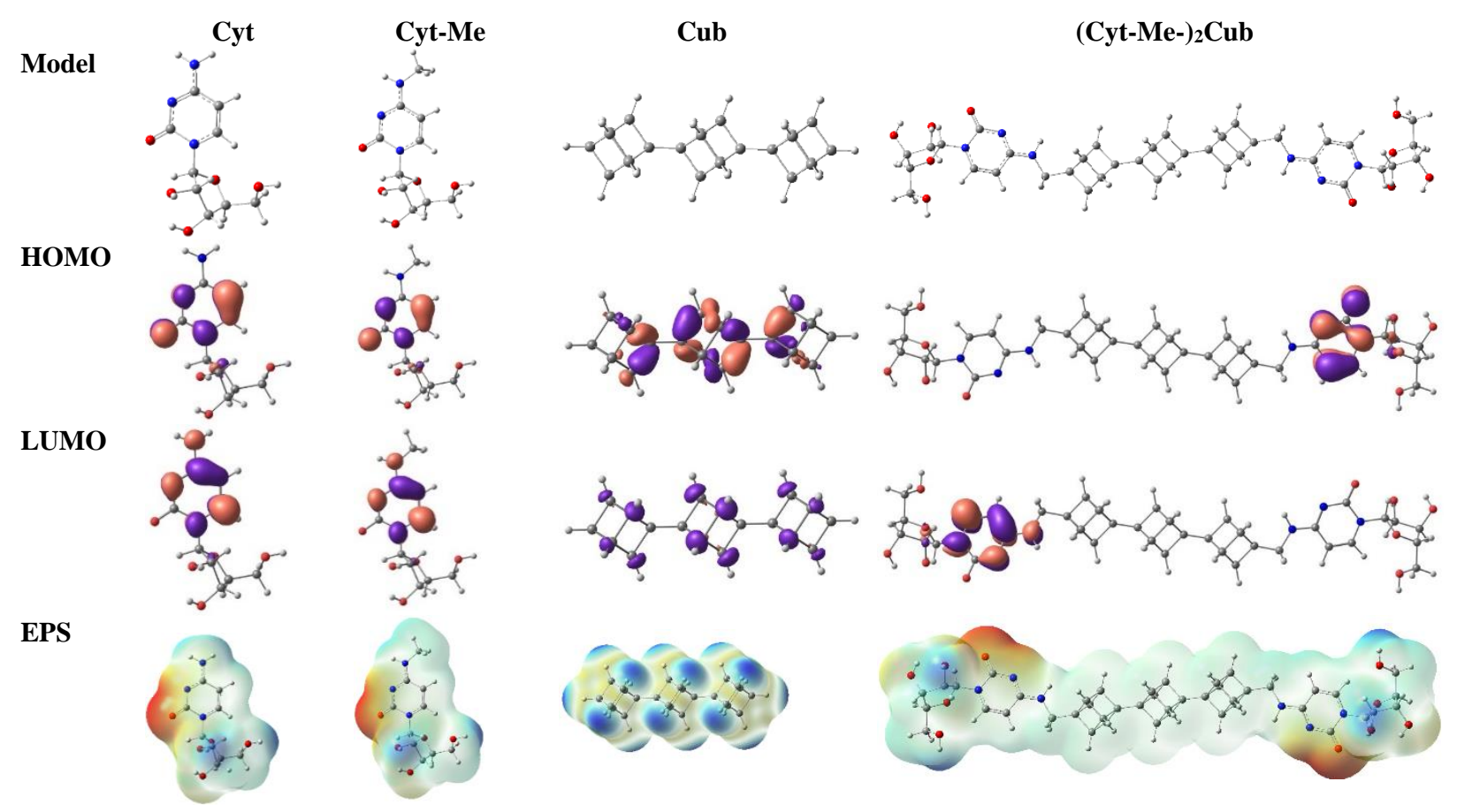

Figure 1. Molecular representations and orbitals.

\begin{tabular}{l|l|l|l|l} 
Property & Cyt & Cyt-Me & Cub & B-Cyt \\
\hline Formula & $\mathrm{C}_{9} \mathrm{H}_{13} \mathrm{~N}_{3} \mathrm{O}_{5}$ & $\mathrm{C}_{10} \mathrm{H}_{15} \mathrm{~N}_{3} \mathrm{O}_{5}$ & $\mathrm{C}_{24} \mathrm{H}_{20}$ & $\mathrm{C}_{44} \mathrm{H}_{46} \mathrm{~N}_{6} \mathrm{O}_{10}$ \\
\hline HOMO & -6.32 & -6.22 & -6.49 & -6.19 \\
\hline LUMO & -1.14 & -1.11 & -0.04 & -1.08 \\
\hline MV cm 3 /mol & 186.87 & 168.532 & 249.569 & 502.981 \\
\hline DM Debye & 9.24 & 9.51 & 0.00 & 7.06 \\
\hline
\end{tabular}

*See Figure 1 for details.

Table 2. Interaction properties*.

\begin{tabular}{l|c|c|c|c} 
Property & Cyt & Cyt-Me & Cub & B-Cyt \\
\hline BE eV & -7.41 & -7.13 & -7.86 & -10.26 \\
\hline RMS & 70.07 & 70.54 & 70.62 & 73.38 \\
\hline AA & HSD41, MET49, & HSD41, MET49, & THR24, THR25, & THR24, THR25, LEU27, \\
& PHE140, LEU141, & TYR54, PHE140, & THR26, LEU27, & HSD41, CYS44, THR45, \\
& ASN142, GLY143, & ASN142, LEU141, & HSD41, MET49, & SER46, MET49, LEU50, \\
& SER144, CYS145, & GLY143, SER144, & ASN142, GLY143, & LEU141, ASN142, \\
& HSD163, HSD164, & CYS145, HSD163, & CYS145, HSD164, & GLY143, SER144, , \\
& MET165, GLU166, & HSD164, MET165, & MET165, GLU166, & CYS145, HSD163, \\
& HSD172, GLN189 & GLU166, ASP187, & GLN189 & HSD164, MET165, \\
& & ARG188, GLN189 & & GLU166, LEU167, \\
& & & & PRO168, GLN189, \\
& & & & THR190, ALA191, \\
& & & & GLN192 \\
\hline
\end{tabular}

\footnotetext{
*See Figure 2 for graphical representations.
} 


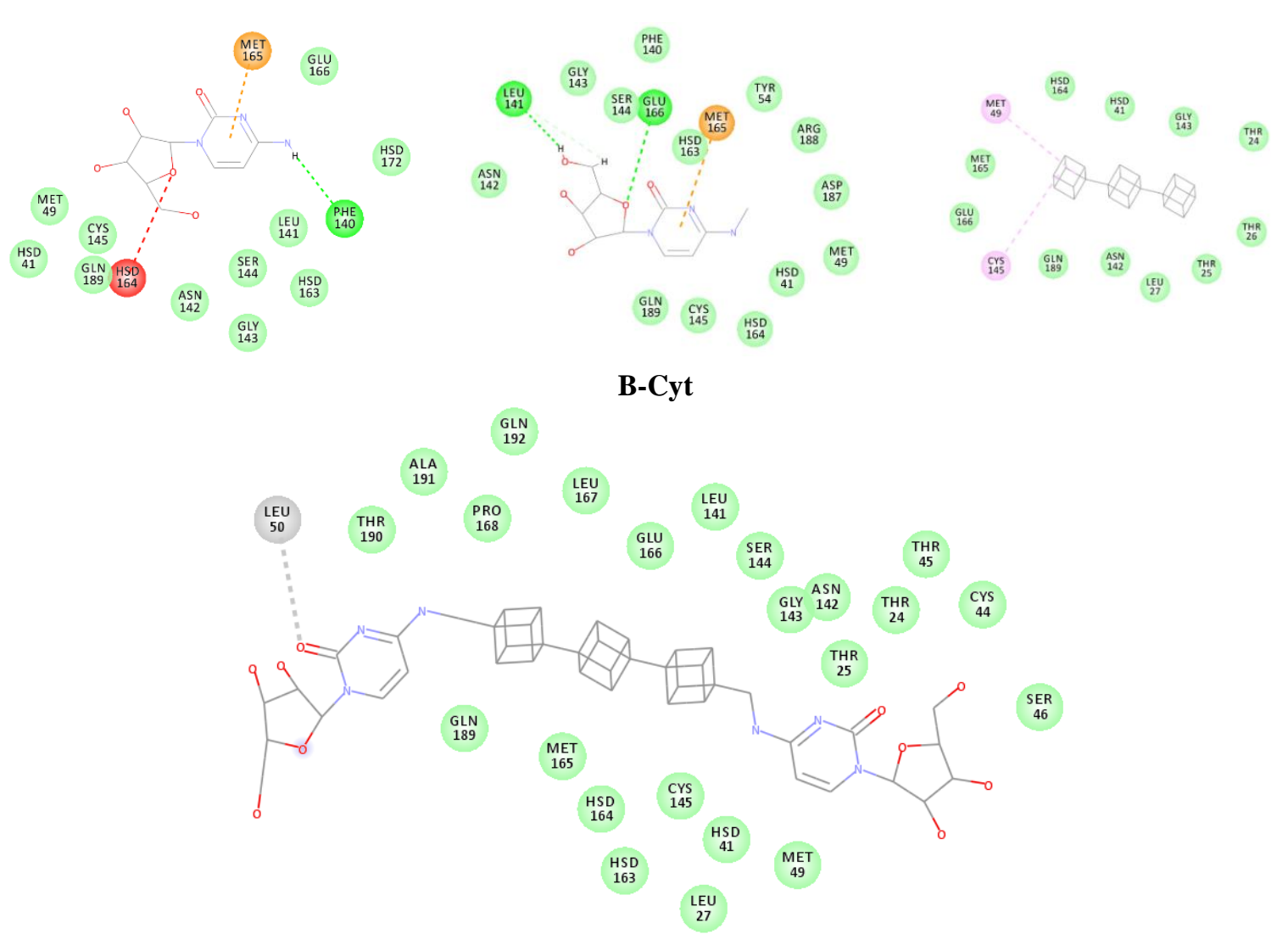

Figure 2. Representations of ligand-target interactions.

\section{Materials and Methods}

3D models of singular molecules of $\mathrm{Cyt}$ and $\mathrm{Cub}$ have been first obtained from ChemSpider Structural Bank [33]. To make the B-Cyt compound's possible formation, the methylated form of Cyt (Cyt-Me) and Cub trimer have been modeled and used to make the BCyt compound. Geometries of all molecular structures have been optimized using the B3LYP/6-31G* density functional theory (DFT) method implemented in the Gaussian program [34]. To examine the global minimization status, frequency calculations have been performed for the optimized structures avoiding the existence of imaginary frequencies for the structures. In the next step, electronic properties of molecular orbitals and electronic distribution have been calculated using the B3LYP/6-31+G* method for the optimized structures in addition to calculating their molar volumes (MV). Dipole moment (DM), the highest occupied, and the lowest unoccupied molecular orbitals (HOMO and LUMO) and electrostatic potentials (ESP) have been obtained for the investigated molecular systems to clarify their original electronic states. In the role of ligand, effects of such molecules were examined on COVID-19 main protease, in the role of a target, by performing molecular docking simulations using the SwissDock web server [35]. In such a process, the best ligand configuration against the target could be detected. The 3D file of COVID-19 main protease has been obtained from Protein Data Bank [36] (ID: 6LU7), and it has been assigned as the target to examine the possibility of ligand...target complex formation. The results of binding energy (BE) and root-mean-square (RMS) have been evaluated for the ligand...target complexes in addition to the interacting amino acids (AA). The idea of forming B-Cyt ligand with possible activity against COVID-19 main protease has been investigated here in silico, and the obtained results were summarized in Tables 1 and 2 and Figures 1 and 2 to be discussed to achieve the purpose of this work. 


\section{Results and Discussion}

Within this in silico work, a new derivative of Cyt has been designed employing the methylated form of original Cyt and a trimer of Cub as linker (Figure 1). The B-Cyt structure has been optimized, and the stabilized model has been obtained, and its verification has been done by frequency calculations avoiding the existence of any imaginary frequencies. Comparing the obtained electronic results (Table 1) for the investigated models of this work could show that the models detect different electronic states, which is very important for determining their activity features. As indicated by HOMO and LUMO, the molecular orbital levels undergo changes, which could yield different electronic behavior for the structures. We expected to have a Cyt derivative with better activity for COVID-19 main protease; here, with the results of Figure 1, the localization of HOMO and LUMO could be seen in different parts of B-Cyt. This trend could mean that the new B-Cyt ligand could play an important role in interactions with enzymes. The HOMO part as the part of electron donor and the LUMO part as the part of electron acceptor is freely localized to have the best chance of interacting with the target. Moreover, ESP could very well approve the dual activity of B-Cyt in comparison with singular Cyt. In this case, the results indicated that the B-Cyt ligand could be expected to work better than the singular Cyt against the target.

The results of docking processes indicated the quantitative values and qualitative interacting AA counterparts. Comparing the values of BE could very well approve the idea of forming a Cyt derivative with more efficient interaction with the target. The values of $\mathrm{BE}$ are very much better for B-Cyt compared with each of singular molecules. Since the B-Cyt volume is larger than each of the singular molecules, it is reasonable that the RMS should be larger than each of the smaller molecules. More interestingly, RMS's value for B-Cyt is slightly larger than each of singular molecules, meaning than B-Cyt is still a good competitor in time to reach the target in competition with the small molecules. The schematic results of Figure 2 indicate that the number of interacting AA were significantly increased in the B-Cyt regarding the singular Cyt ligand. More interestingly, common AA is seen in both complexes meaning that the B-Cyt could interact as the original Cyt site but with better efficacy against the target. The role of Cub is very much important, in which it helped the Cyt to have better interactions with the target. It is important to note that Cyt is used as an anticancer or anti-HIV for the patients, and they could be protected against the COVID-19 by consuming a B-Cyt. Although the drugs should be specific for curing diseases, a dual activity could make the pharmaceutical compounds more potent agents for treating such unwanted diseases like COVID-19. For such a current situation of unknown treatments for the COVID-19 pandemic, it is unavoidable to investigate new pharmaceutical compounds for this purpose. Working on already known compounds such as Cyt with potent activity against enzymes is also very important, as they could be considered lead compounds to generate such B-Cyt derivative.

\section{Conclusions}

This in silico work has been done to investigate B-Cyt's formation as a new Cyt derivative to examine its interaction with COVID-19 main protease. The formation of such a derivative could be possible within the obtained results because of its stabilization without imaginary frequency by DFT methods. The obtained orbital-based electronic properties indicated that B-Cyt's structure could provide better sites for interactions with the target. In other parts, ligand-target interactions results indicated that the new ligand B-Cyt could interact 
with the target COVID-19 main protease very much better than the original Cyt. Better value of $\mathrm{BE}$ and a larger AA number are all the benefits of B-Cyt versus original Cyt to be consumed against the unwanted COVID-19 pandemic.

\section{Funding}

This research received no external funding.

\section{Acknowledgments}

This research has no acknowledgment.

\section{Conflicts of Interest}

The authors declare no conflict of interest.

\section{References}

1. Jones, P.A.; Taylor, S.M. Cellular differentiation, cytidine analogs and DNA methylation. Cell 1980, 20, 8593, https://doi.org/10.1016/0092-8674(80)90237-8.

2. Baswan, S.M.; Leverett, J.; Pawelek, J. Clinical evaluation of the lightening effect of cytidine on hyperpigmented skin. J Cosmetic Dermatology 2019, 18, 278-285, https://doi.org/10.1111/jocd.12784.

3. Faramarzi. R.; Falahati, M.; Mirzaei, M. Interactions of fluorouracil by CNT and BNNT: DFT analyses. Advanced Journal of Science and Engineering 2020, 1, 62-66, https://doi.org/10.22034/AJSE.2012062.

4. Ghamsari, P.A.; Samadizadeh, M.; Mirzaei, M. Halogenated derivatives of cytidine: Structural analysis and binding affinity. Journal of Theoretical and Computational Chemistry 2020, 19, https://doi.org/10.1142/S0219633620500339.

5. Zhang, W.; Pal, A.; Ricardo, A.; Szostak, J.W. Template-directed nonenzymatic primer extension using 2methylimidazole-activated morpholino derivatives of guanosine and cytidine. Journal of the American Chemical Society 2019, 141, 12159-12166, https://doi.org/10.1021/jacs.9b06453.

6. Teppang, K.L.; Lee, R.W.; Burns, D.D.; Turner, M.B.; Lokensgard, M.E.; Cooksy, A.L.; Purse, B.W. Electronic modifications of fluorescent cytidine analogues control photophysics and fluorescent responses to base stacking and pairing. Chemistry-A European Journal 2019, 25, 1249-1259, https://doi.org/10.1002/chem.201803653.

7. Li, X.; Erturk, E.; Chen, X.; Kumar, S.; Guo, C.; Jockusch, S.; Russo, J.J.; Bestor, T.H.; Juet, J. Photochemical conversion of a cytidine derivative to a thymidine analog via [2+2]-cycloaddition. Photochemical \& Photobiological Sciences 2018, 17, 1049-1055, https://doi.org/10.1039/C8PP00161H.

8. Bhattacharyya, T.; Chaudhuri, R.; Das, K.S.; Mondal, R.; Mandal, S.; Dash, J. Cytidine-derived hydrogels with tunable antibacterial activities. ACS Applied Bio Materials 2019, 2, 3171-3177, https://doi.org/10.1021/acsabm.9b00438.

9. Gowher, H.; Jeltsch, A. Mechanism of inhibition of DNA methyltransferases by cytidine analogs in cancer therapy. Cancer Biology \& Therapy 2004, 3, 1062-1068, https://doi.org/10.4161/cbt.3.11.1308.

10. Derache, A.; Iwuji, C.C.; Danaviah, S.; Giandhari, J.; Marcelin, A.G.; Calvez, V.; de Oliveira, T.; Dabis, F.; Pillay, D.; Gupta, R.K. Predicted antiviral activity of tenofovir versus abacavir in combination with a cytosine analogue and the integrase inhibitor dolutegravir in HIV-1-infected South African patients initiating or failing first-line ART. Journal of Antimicrobial Chemotherapy 2019, 74, 473-479, https://doi.org/10.1093/jac/dky428.

11. Faraj, A.; Agrofoglio, L.A.; Wakefield, J.K.; McPherson, S.; Morrow, C.D.; Gosselin, G.; Mathe, C.; Imbach, J.L.; Schinazi, R.F; Sommadossi J.P. Inhibition of human immunodeficiency virus type 1 reverse transcriptase by the 5'-triphosphate beta enantiomers of cytidine analogs. Antimicrobial Agents and Chemotherapy 1994, 38, 2300-2305, https://doi.org/10.1128/AAC.38.10.2300.

12. Lin, T.S.; Luo, M.Z.; Liu, M.C. A stereospecific synthesis of $2^{\prime}, 3^{\prime}$-dideoxy- $\beta$-1-cytidine ( $\beta$-1-ddC), a potent inhibitor against human hepatitis B virus (HBV) and human immunodeficiency virus (HIV). Tetrahedron Letters 1994, 35, 3477-3480, https://doi.org/10.1016/S0040-4039(00)73214-X.

13. Camiener, G.W. Studies of the enzymatic deamination of ara-cytidine-V: Inhibition in vitro and in vivo by tetrahydrouridine and other reduced pyrimidine nucleosides. Biochemical Pharmacology 1968, 17, 19811991, https://doi.org/10.1016/0006-2952(68)90114-7.

14. McPartland, R.P.; Wang, M.C.; Bloch, A.; Weinfeld, H. Cytidine 5'-triphosphate synthetase as a target for inhibition by the antitumor agent 3-deazauridine. Cancer Research 1974, 34, 3107-3111. 
15. Spinelli, A.; Pellino, G. COVID-19 pandemic: perspectives on an unfolding crisis. The British Journal of Surgery 2020, 107, 785-787, https://doi.org/10.1002/bjs.11627.

16. Harismah, K.; Mirzaei, M. COVID-19: a serious warning for emergency health innovation. Advanced Journal of Science and Engineering 2020, 1, 32-33, https://doi.org/10.22034/AJSE.2012032.

17. Gates, B. Responding to Covid-19-a once-in-a-century pandemic?. New England Journal of Medicine 2020, 382, 1677-1679, https://doi.org/10.1056/NEJMp2003762.

18. Rome, B.N.; Avorn, J. Drug evaluation during the Covid-19 pandemic. New England Journal of Medicine 2020, 382, 2282-2284, https://doi.org/10.1056/NEJMp2009457.

19. Wang, J. Fast identification of possible drug treatment of coronavirus disease-19 (COVID-19) through computational drug repurposing study. Journal of Chemical Information and Modeling 2020, 6, 3277-3286, https://doi.org/10.1021/acs.jcim.0c00179.

20. Mirzaei, M.; Harismah, K.; Da'I, M.; Salarrezaei, E.; Roshandel, Z. Screening efficacy of available HIV protease inhibitors on COVID-19 protease. Journal Military Medicine 2020, 22, 100-107, https://doi.org/10.30491/JMM.22.2.100.

21. Harismah, K.; Mirzaei, M. Favipiravir: Structural analysis and activity against COVID-19. Advanced Journal of Chemistry B 2020, 2, 55-60, https://doi.org/10.33945/SAMI/AJCB.2020.2.3.

22. Qamar, M.T.; Alqahtani, S.M.; Alamri, M.A.; Chen, L.L. Structural basis of SARS-CoV-2 3CLpro and antiCOVID-19 drug discovery from medicinal plants. Journal of Pharmaceutical Analysis 2020, 10, 313-319, https://doi.org/10.1016/j.jpha.2020.03.009.

23. Biegasiewicz, K.F.; Griffiths, J.R.; Savage, G.P.; Tsanaktsidis, J.; Priefer, R. Cubane: 50 years later. Chemical Reviews 2015, 115, 6719-6745, https://doi.org/10.1021/cr500523x.

24. Houston, S.D.; Fahrenhorst-Jones, T.; Xing, H.; Chalmers, B.A.; Sykes, M.L.; Stok, J.E.; Soto, C.F.; Burns, J.M.; Bernhardt, P.V.; De Voss, J.J.; Boyle, G.M.; Smith, M.T.; Tsanaktsidis, J.; Savage, G.P.; Avery, V.M.; Williams, C.M. The cubane paradigm in bioactive molecule discovery: further scope, limitations and the cyclooctatetraene complement. Organic \& Biomolecular Chemistry 2019, 17, 6790-6798, https://doi.org/10.1039/C9OB01238A.

25. Katin, K.P.; Maslov, M.M. Chemical functionalization effects on cubane-based chain electronic transport. Advances in Condensed Matter Physics 2015, 2015, https://doi.org/10.1155/2015/754873.

26. Harismah, K.; Mirzaei, M. In silico interactions of steviol with monoamine oxidase enzymes. Lab-in-Silico 2020, 1, 3-6.

27. Mirzaei, M. Science and engineering in silico. Advanced Journal of Science and Engineering 2020, 1, 1-2, https://doi.org/10.22034/AJSE2011001.

28. Harismah, K.; Mirzaei, M. Steviol and iso-steviol vs. cyclooxygenase enzymes: in silico approach. Lab-inSilico 2020, 1, 11-15.

29. Behzadi, H.; Hadipour, N.L.; Mirzaei, M. A density functional study of $17 \mathrm{O}, 14 \mathrm{~N}$ and $2 \mathrm{H}$ electric field gradient tensors in the real crystalline structure of $\alpha$-glycine. Biophysical Chemistry 2007, 125, 179-183, https://doi.org/10.1016/j.bpc.2006.07.010.

30. Samadi, Z.; Mirzaei, M.; Hadipour, N.L.; Khorami, S.A. Density functional calculations of oxygen, nitrogen and hydrogen electric field gradient and chemical shielding tensors to study hydrogen bonding properties of peptide group (OC-NH) in crystalline acetamide. Journal of Molecular Graphics and Modelling 2008, 26, 977-981, https://doi.org/10.1016/j.jmgm.2007.08.003.

31. Durojaiye, A.B.; Clarke, J.R.; Stamatiades, G.A.; Wang, C. Repurposing cefuroxime for treatment of COVID-19: a scoping review of in silico studies. Journal of Biomolecular Structure and Dynamics. 2020, in press, https://doi.org/10.1080/07391102.2020.1777904.

32. Khalid, H.; Hussain, R.; Hafeez, A. Virtual screening of piperidine based small molecules against COVID19. Lab-in-Silico 2020, 1, 50-55.

33. Pence, H.E.; Williams, A. ChemSpider: an online chemical information resource. Journal of Chemical Education 2010, 87, 1123-1124, https://doi.org/10.1021/ed100697w.

34. Frisch, M.J.; Trucks, G.W.; Schlegel, H.B.; Scuseria, G.; Robb, M.; Cheeseman, J.; Scalmani, G.; Barone, V.; Mennucci, B.; Petersson, G. Gaussian 09, Revision D.01, Gaussian. Inc., Wallingford CT. 2009.

35. Bitencourt-Ferreira, G. Docking with SwissDock. Methods in Molecular Biology 2019, 2053, 189-202, https://doi.org/10.1007/978-1-4939-9752-7_12.

36. Burley, S.K.; Berman, H.M.; Bhikadiya, C.; Bi, C.; Chen, L.; Di Costanzo, L.; Christie, C.; Dalenberg, K.; Duarte, J.M.; Dutta, S.; Feng, Z.; Ghosh, S.; Goodsell, D.S.; Green, R.K.; Guranović, V.; Guzenko, D.; Hudson, B.P.; Kalro, T.; Liang, Y.; Lowe, R.; Namkoong, H.; Peisach, E.; Periskova, I.; Prlić, A.; Randle, C.; Rose, A.; Rose, P.; Sala, R.; Sekharan, M.; Shao, C.; Tan, L.; Tao, Y.-P.; Valasatava, Y.; Voigt, M.; Westbrook, J.; Woo, J.; Yang, H.; Young, J.; Zhuravleva, M.; Zardecki, C. RCSB protein data bank: biological macromolecular structures enabling research and education in fundamental biology, biomedicine, biotechnology and energy. Nucleic Acids Research 2019, 47, 464-474, https://doi.org/10.1093/nar/gky1004. 